\title{
Some non-linear function theoretic properties of Riemannian manifolds
}

\author{
Stefano Pigola, Marco Rigoli and Alberto G. Setti
}

Dedicated to the memory of Franca Burrone Rigoli

\begin{abstract}
We study the appropriate versions of parabolicity stochastic completeness and related Liouville properties for a general class of operators which include the $p$-Laplace operator, and the non linear singular operators in non-diagonal form considered by J. Serrin and collaborators.
\end{abstract}

\section{Introduction}

The starting point of the present note is the circle of ideas in classical potential theory, which relate the parabolicity and stochastic completeness of a manifold on one hand, and their function theoretic counterparts on the other, and in particular results due to Khas'minskii which provide sufficient conditions for a manifold to be parabolic, respectively stochastically complete. These ideas allow also to establish comparison theorems with model manifolds in the sense of Greene and Wu under curvature conditions.

It was recently shown by the authors that the parabolicity/stochastic completeness of a manifold may be described in terms of suitable versions of a global weak maximum principle. Explicitly, $M$ is parabolic (resp. stochastically complete) if and only if for every $u \in C^{2}(M), u$ bounded above, and for every $\eta>0$ we have

$$
\inf _{\{u>\sup u-\eta\}} \Delta u<0 \quad(\text { resp. } \leq 0)
$$

(see [17] and [18]).

2000 Mathematics Subject Classification: 31C12, 53C21, 58J05.

Keywords: Non-linear potential theory, $p$-Laplacian type operators, Riemannian manifolds. 
The note grew out in an attempt to extend these ideas in the non-linear setting of the potential theory of the $p$-laplacian. Indeed, the link between parabolicity and stochastic completeness and weak maximum principle allows us to deal with a more general class of nonlinear operators modelled on the $p$-Laplacian. We are able to treat divergence form operators like the $\varphi$-Laplacian or even the non-diagonal operators studied in Euclidean setting by J. Serrin and collaborators, essentially under the assumption of the solvability of the corresponding Dirichlet problem. We point out in this respect that the solvability of the Dirichlet problem plays an essential role even in the classical potential theory of the $p$-Laplacian.

Throughout the paper we shall denote with $\mathcal{L}_{\varphi, h}$ the operator defined thus:

Let $h$ be a positive definite symmetric covariant tensor field defined on $M$, that is a positive definite section of the bundle $S^{2} T^{*} M$ of symmetric bilinear forms on $T M$. Let also $\varphi \in C^{1}((0,+\infty)) \cap C^{0}([0,+\infty))$ be a function satisfying the following structural conditions

$$
\begin{aligned}
& \text { (i) } \varphi(0)=0, \quad\left(\text { ii) } \varphi(t)>0, \quad \text { (iii) } \varphi(t) \leq A t(\kappa+t)^{p-2},\right. \\
& \text { (iv) } \frac{\varphi(t)}{t} h(\xi, \xi)+\left(\varphi^{\prime}(t)-\frac{\varphi(t)}{t}\right)\langle v, \xi\rangle h(v, \xi)>0
\end{aligned}
$$

for some constants $A>0, \kappa \geq 0, p>1$, and for every $t>0$ and $v, \xi \in T M$ with $|v|=|\xi|=1$. Observe that the ellipticity condition (0.1) (iv), which will be used to prove a version of a comparison result valid in our context (see Section 1 below), implies that

$$
\varphi^{\prime}(t)>0 \quad \forall t>0 .
$$

and it is in fact equivalent to the latter condition when $h=h(x)\langle$,$\rangle is a$ conformal deformation of the metric.

For $u \in C^{0}(M) \cap W_{l o c}^{1, p}(M)$ let $\mathcal{L}_{\varphi, h} u$ be defined, in the appropriate weak sense, by

$$
\mathcal{L}_{\varphi, h} u=\operatorname{div}\left(|\nabla u|^{-1} \varphi(|\nabla u|) h(\nabla u, \cdot)^{\sharp}\right),
$$

where $^{\sharp}: T^{*} M \rightarrow T M$ denotes the musical isomorphism, so that $h(\nabla u, \cdot)^{\sharp}$ is the vector field on $M$ defined by

$$
\left\langle h(\nabla u, \cdot)^{\sharp}, X\right\rangle=h(\nabla u, X), \quad \forall X \in T_{x} M .
$$

It should be observed that in order to define $\mathcal{L}_{\varphi, h} u$ for $u \in C^{1}(M)$ we may dispense with conditions (0.1) (iii) and (iv).

The operators $\mathcal{L}_{\varphi, h}$ may be viewed as the natural, intrinsic generalization to Riemannian manifolds of the fully quasilinear singular elliptic operators considered by Pucci Serrin and Zou (see [21], [19], [20]). 
They also generalize the $\mathcal{A}$-Laplace operators as defined in [7] in the setting of nonlinear potential theory. For the latter class of operators we refer to work by I. Holopainen, [9], who obtains interesting Liouville type results. From a somewhat different point of view, see also the recent paper by T. Coulhon, I. Holopainen, and L. Saloff-Coste [2].

Note that if we choose $h$ to be the metric tensor of $M$, then the operator $\mathcal{L}_{\varphi, h}$ reduces to the $\varphi$-Laplacian $\mathcal{L}_{\varphi}$, which in turn, for appropriate choices of the function $\varphi$ leads to well known operators such as:

- the Laplace-Beltrami operator, corresponding to $\varphi(t)=t$;

- the $p$-Laplacian, div $\left(|\nabla u|^{p-2} \nabla u\right)$ corresponding to $\varphi(t)=t^{p-1}, p>1$;

- the mean curvature operator $\operatorname{div}\left(\frac{\nabla u}{\sqrt{1+|\nabla u|^{2}}}\right)$, corresponding to $\varphi(t)=$ $t\left(1+t^{2}\right)^{-1 / 2}$.

While many of our results hold for the general class of operators defined above, the full strength of our results depend on the solvability of the Dirichlet problem and the validity of appropriate regularity results. We therefore consider the following additional conditions on $h$ and $\varphi$ :

$$
h_{-}\langle,\rangle \leq h \leq h_{+}\langle,\rangle,
$$

in the sense of quadratic forms, for some positive constants $h_{-}, h_{+}$, and

$$
\begin{aligned}
& \text { (v) } \frac{\varphi(s)}{s} h(\xi, \xi)+\left(\varphi^{\prime}(s)-\frac{\varphi(s)}{s}\right)\langle v, \xi\rangle h(v, \xi) \geq \gamma(\kappa+s)^{p-2} \\
& \text { (vi) }\left|\frac{\varphi(s)}{s} h\left(\xi, \xi^{\prime}\right)+\left(\varphi^{\prime}(s)-\frac{\varphi(s)}{s}\right)\langle v, \xi\rangle h\left(v, \xi^{\prime}\right)\right| \leq \Gamma(\kappa+s)^{p-2},
\end{aligned}
$$

for some constants $\gamma, \Gamma>0, \kappa \geq 0$, and every $s>0, \xi, \xi^{\prime}, v \in T_{x} M$ with $|\xi|=\left|\xi^{\prime}\right|=|v|=1$. The above conditions on $h$ and $\varphi$ guarantee that the operator $\mathcal{L}_{\varphi, h}$ satisfies the main hypotheses considered in the papers by T. Kura, [12], and P. Tolksdorf, [26], and imply that the method of super-sub solution is applicable to find solutions of the Dirichelt problem associated to the equation $\mathcal{L}_{\varphi, h} u=q(x) u$, and that the relative solutions are of class $C^{1}$.

The prototypical example of operators satisfying condition (0.1) (i)-(vi), and $(0.3)$ is of course the $p$-Laplacian (with $h()=,\langle$,$\rangle ), or the \mathcal{A}$-Laplacian considered in [7] and [8]. We stress however that we do not impose homogeneity condition

$$
\mathcal{A}_{x}(t \xi)=t^{p-1} \mathcal{A}_{x}(\xi) \quad \forall \xi \in T_{x}(M), t>0,
$$

which is assumed by those authors, and which plays a crucial role in the capacity approach they follow. As a consequence of this, we are forced to use entirely different techniques. 
As a further example, note that if the tensor $h$ is a multiple of the metric, that is, $h=h(x)\langle$,$\rangle , the above conditions read$

$$
\begin{aligned}
& (\mathrm{v})^{\prime} \quad \min \left(\varphi^{\prime}(s), \frac{\varphi(s)}{s}\right) \geq \gamma(\kappa+s)^{p-2} \\
& (\mathrm{vi})^{\prime} \quad \max \left(\varphi^{\prime}(s), \frac{\varphi(s)}{s}\right) \leq \Gamma(\kappa+s)^{p-2} .
\end{aligned}
$$

We next introduce the notation and terminology that will be used in the rest of the paper. In the sequel $\lambda$ will be a fixed nonnegative real number.

Definition 0.1. A function $u \in C^{0}(M) \cap W_{l o c}^{1, p}(M)$ is said to be $\lambda$-subharmonic with respect to $\mathcal{L}_{\varphi, h}$ if $\mathcal{L}_{\varphi, h} u \geq \lambda u$. If the opposite inequality holds, or the inequality is replaced by equality we will say that $u$ is $\lambda$-superharmonic, $\lambda$-harmonic, respectively. In all cases the (in)equalities are understood to hold in the appropriate weak sense.

Definition 0.2. We say that the $\lambda$-Liouville property for $C^{1}(M)$ (respectively, for $C^{0}(M) \cap W_{l o c}^{1, p}(M)$ functions is valid for the operator $\mathcal{L}_{\varphi, h}$ if, every $u \in C^{1}(M)$ (respectively $u \in C^{0}(M) \cap W_{l o c}^{1, p}(M)$ ), bounded above, $\lambda$-subharmonic, and nonnegative in case $\lambda>0$, is constant on $M$ (and therefore identically zero if $\lambda>0$ ).

Definition 0.3. We say that the $\lambda$-weak maximum principle at infinity for $C^{1}$ (respectively, for $C^{0} \cap W_{l o c}^{1, p}$ ) functions holds for the operator $\mathcal{L}_{\varphi, h}$ if for every non constant function $u \in C^{1}(M)$ (respectively, $u \in C^{0}(M) \cap$ $\left.W_{l o c}^{1, p}(M)\right)$ with $u^{*}=\sup _{M} u<+\infty$, and for every $\eta<u^{*}$, we have

$$
\inf _{\Omega_{\eta}} \mathcal{L}_{\varphi, h} u \begin{cases}<0 & \text { if } \lambda=0 \\ \leq 0 & \text { if } \lambda>0\end{cases}
$$

where we have set $\Omega_{\eta}=\{x: u(x)>\eta\}$.

In the above definition, the inequality is understood in distributional sense. Explicitly, we say that $\inf _{\Omega} \mathcal{L}_{\varphi, h} u<C$ if there exists $0 \leq \psi \in C_{c}^{\infty}(\Omega)$ such that

$$
\int_{\Omega}|\nabla u|^{-1} \varphi(|\nabla u|) h(\nabla u, \nabla \psi)<C \int_{\Omega} \psi
$$

and that $\inf _{\Omega} \mathcal{L}_{\varphi, h} u \leq C$ if, for every $\epsilon>0, \inf _{\Omega} \mathcal{L}_{\varphi, h} u<C+\epsilon$.

It is straightforward to check that when $u \in C^{2}$ is such that $\mathcal{L}_{\varphi, h} u \in C^{0}$, then the weak inequality holds if and only if

$$
\inf _{x \in \Omega} \mathcal{L}_{\varphi, h} u(x)<C \quad(\text { resp. } \quad \leq C) .
$$


In this case, the condition above can be reformulated as follows:

There exists a sequence $\left\{x_{k}\right\} \subset M$ such that

$$
\begin{aligned}
& \text { (i) } u\left(x_{k}\right)>u^{*}-\frac{1}{k} \\
& \text { (ii) } \mathcal{L}_{\varphi, h} u\left(x_{k}\right) \begin{cases}<0 & \text { if } \lambda=0 \\
<1 / k & \text { if } \lambda>0 .\end{cases}
\end{aligned}
$$

Note that (0.7) is part of the conditions that enter in the definition of the Omori-Yau maximum principle, where however the extra condition that $|\nabla u|$ tends to zero is imposed. Indeed, the expression weak maximum principle was adopted in [17] and [18] to stress the fact that no condition on $\nabla u$ is required. We also remark that, for $\lambda>0$ the $\lambda$-weak maximum principle is equivalent to the following property (see, [17], [18]):

For any function $f \in C^{0}(\mathbb{R})$, and any bounded above function $u$ satisfying the appropriate regularity condition

$$
\text { if } \mathcal{L}_{\varphi, h} u \geq f(u) \text { on } \Omega_{\eta}=\left\{x: u(x)>u^{*}-\eta\right\}, \quad \text { then } f\left(u^{*}\right) \leq 0 .
$$

Definition 0.4. We say that the $\lambda$-Khas'minskii test for the operator $\mathcal{L}_{\varphi, h}$ is valid on $M$ if there exists a compact set $K$ in $M$, such that, for every $x_{o} \in M \backslash K$, and every $\epsilon>0$, there exists a function $\gamma=\gamma_{x_{o}, \epsilon} \in C^{0}(M \backslash K)$ $\cap W_{\text {loc }}^{1, p}(M \backslash K)$ such that

$$
\begin{array}{ll}
\text { (i) } \gamma>0 \text { on } M \backslash K, & \text { (ii) } \gamma\left(x_{o}\right)<\epsilon, \\
\text { (iii) } \gamma(x) \rightarrow+\infty \text { as } x \rightarrow \infty, & \text { (iv) } \mathcal{L}_{\varphi, h} \gamma \leq \lambda \gamma \text { on } M \backslash K
\end{array}
$$

It is worth noting that when the operator satisfies the homogeneity condition (0.4) with $p \geq 2$, then the $\lambda$-Khas'minskii test is valid provided there exists a function $\gamma_{o}$ defined in the complement of a compact set $K$ such that

$$
\gamma_{o}(x) \rightarrow+\infty \text { as } x \rightarrow+\infty, \quad \text { and } \quad \mathcal{L}_{\varphi, h} \gamma_{o} \leq \lambda \gamma_{o} \text { in } M \backslash K,
$$

This is the usual formulation of the $\lambda$-Khas'minskii test for the Laplacian (see [5]), and to see that it implies all the conditions listed above, note first that, by adding a suitable constant, we may assume that $\gamma_{o}$ is nonnegative. Further, since $p \geq 2$, given $x_{o} \in M \backslash K$ and $\epsilon>0$, we can multiply $\gamma_{o}$ by a constant $\alpha$ small enough that $\alpha \gamma\left(x_{o}\right)<\epsilon$, and

$$
\mathcal{L}_{\varphi, h}\left(\alpha \gamma_{o}\right)=\alpha^{p-1} \mathcal{L}_{\varphi, h} \gamma_{o} \leq \alpha^{p-2} \lambda\left(\alpha \gamma_{o}\right) \leq \lambda\left(\alpha \gamma_{o}\right) \quad \text { in } M \backslash K .
$$

In the case of the Laplace operator, one can use the solvability of the Dirichlet problem, and standard regularity results, to show that if the $\lambda$-Liovuille 
property holds for $C^{1}$, indeed even $C^{\infty}$, functions, then it holds for $C^{0} \cap W_{l o c}^{1, p}$ functions. Moreover, it is well known, see e.g. the very informative survey article by A. Grigor'yan [5], that the $\lambda$-Liouville property is equivalent to the parabolicity (if $\lambda=0$ ), respectively stochastic completeness (if $\lambda>0$ ) of the underlying manifold, that the $\lambda$-Khas'minshii test implies the $\lambda$-Liouville property, and, that the parabolicity of the manifold implies the 0-Khas'minskii test (see, [16] and [24]). To conclude this chain of equivalences, it was recently shown by the present authors ([17], [18]) that the $\lambda$-Liouville property is in fact equivalent to the validity of the $\lambda$-weak maximum principle. Using this fact one can give a direct proof of the fact that the $\lambda$-Khas'minskii test implies the validity of $\lambda$-Liouville property.

Many of these implications have been extended to more general, nonlinear operators, like $p$-Laplacian, see e.g. [9], and the $\varphi$-Laplacian, [23], where the parabolicity is defined in term of the validity of the 0-Liouville property for the appropriate operator. Indeed, the 0-Liouville property for the $p$-Laplacian is equivalent to other properties like non-existence of the positive Green function, which serve as definition of parabolicity in linear potential theory.

Similarly, the validity of the $\lambda$-Liouville property with $\lambda>0$ may be taken as definition of stochastic completeness relative to the operator under consideration.

Our first main result is the following.

Theorem A. Assume that $h$ and $\varphi$ satisfy conditions (0.3) and (0.1) (i)(vi), respectively, and let $\lambda \geq 0$. Then the following properties are equivalent:

(i) The $\lambda$-weak maximum principle holds for $C^{0}(M) \cap W_{\text {loc }}^{1, p}(M)$ functions;

(ii) The $\lambda$-weak maximum principle holds for $C^{1}(M)$ functions;

(iii) The operator $\mathcal{L}_{\varphi, h}$ has $\lambda$-Liouville property for $C^{1}(M)$ functions;

(iv) The operator $\mathcal{L}_{\varphi, h}$ has $\lambda$-Liouville property for $C^{0}(M) \cap W_{\text {loc }}^{1, p}(M)$ functions.

Furthermore, if the $\lambda$-Khas'minnskii test is valid, then the $\lambda$-weak maximum principle for $C^{1}$ functions holds.

As mentioned above some of the implications hold if we assume that the function $\varphi$ satisfies only assumptions (0.1) (i)-(iv). The proofs in Section 2 below are organized in such a way as to show which implications are valid in this greater generality, and where the extra assumptions are needed.

Our second main result is a comparison result with models for the parabolicity/stochastic completeness of a manifold with respect to the $\varphi$-Laplacian, which extends to this class of operators results valid for the Laplacian 
(see [5]). Before stating the result, we recall the definition of a model manifold in the sense of Greene and $\mathrm{Wu}([4]):$ Let $\sigma:[0,+\infty) \rightarrow[0,+\infty)$ be a smooth function satisfying $\sigma(0)=0, \sigma(t)>0$ for $t>0, \sigma^{\prime}(0)=1$, $\sigma^{(2 k)}(0)=0$ for every $k=0,1, \ldots$, the $m$-dimensional model defined by $\sigma$ is the manifold $\left(\tilde{M}, \widetilde{\langle,\rangle_{\sigma}}\right)$, where $\tilde{M}=\mathbb{R}^{m}$ with the metric defined in polar coordinates $(r, \theta)$ by $\widetilde{\langle,\rangle_{\sigma}}=d r^{2}+\sigma(r)^{2} d \theta^{2}$, where $d \theta^{2}$ denotes the canonical metric of the unit sphere $\mathbb{S}^{m-1}$ in $\mathbb{R}^{m}$. Note that the requests on $\sigma$ enable us to extend the metric smoothly on all of $\tilde{M}$. The condition that the derivatives of even orders vanish in 0 does not appear in [4], but, as shown in [11], it is needed to guarantee the smoothness of the resulting metric.

Theorem B. Let $(M,\langle\rangle$,$) be a complete Riemannian manifold, let o \in M$ and denote by $r(x)$ the Riemannian distance function from o, and by cut $(o)$ the cut locus of o. Assume that the function $\varphi$ satisfies (0.1) (i)-(iv), and suppose also that

$$
\phi(t) \rightarrow+\infty \quad \text { as } t \rightarrow+\infty \text {. }
$$

(i) Assume that the differential inequality

$$
\Delta r(x) \leq(m-1) \frac{\sigma^{\prime}}{\sigma}(r(x))
$$

holds in $M \backslash(\{o\} \cap \operatorname{cut}(o))$, where $\sigma$ is a smooth function satisfying the conditions listed in the definition of model manifold. If the $\lambda$-weak maximum principle for the operator $\tilde{\mathcal{L}}_{\varphi}$ holds on $\left(\tilde{M}, \widetilde{\langle,\rangle_{\sigma}}\right)$, then it also holds for the operator $\mathcal{L}_{\varphi}$ on $M$.

(ii) Assume that $o$ is a pole of $M$, that the differential inequality

$$
\Delta r(x) \geq(m-1) \frac{\sigma^{\prime}}{\sigma}(r(x))
$$

holds in $M \backslash\left\{\right.$ o\}. If the $\lambda$-weak maximum principle for the operator $\mathcal{L}_{\varphi}$ holds on $M$, then it also holds for $\tilde{\mathcal{L}_{\varphi}}$ on $\tilde{M}$.

In Section 1 we prove the various implications, which lead to a proof of Theorem A. We then consider the case of models, and obtain a necessary and sufficient condition, expressed in terms of volume growth, for a model manifold to satisfy the $\lambda$-Khas'minskii test for the $\varphi$-Laplacian. Using this we obtain a comparison result for the $\mathcal{L}_{\varphi}$-parabolicitiy, resp. stochastic completeness of general Riemannian manifolds. We then extend some of the above considerations to the case of the operator $\mathcal{L}_{\varphi, h}$, and describe a sufficient condition, expressed in terms of curvature, that the $\lambda$-Khas'minskii test 
for $\mathcal{L}_{\varphi, h}$ holds. We end the paper discussing a problem raised by Holopainen, concerning the vanishing of $p$-superharmonic functions belonging to $L^{p-1}$, and which we will be referring to as the $L^{p-1}$-Liouville property. It is easy to see that, for the ordinary Laplacian, the $L^{1}$-Liouville property is implied by the stochastic completeness of the manifold, and that this is in fact an equivalence in the case of models. One is therefore led to expect that the validity of the $L^{p-1}$-Liouville property for the $p$-Laplacian may be related to the version of $p$-stochastic completeness defined in terms of $\lambda$-Liouville property. We investigate this relationship in the case of model manifolds, and exploiting the characterization of stochastic completeness of models in terms of volume growth, we show that $p$-stochastic completeness indeed implies the validity of the $L^{p-1}$-Liouville property if $p \leq 2$, while the reverse implication holds if $p \geq 2$. We then show, adapting an example of Holopainen that neither of the two implications can be reversed if $p \neq 2$.

\section{Proof of Theorem A and related facts}

We begin by collecting some general facts on the operator $\mathcal{L}_{\varphi, h}$. In particular, we prove a comparison principle valid in our context, and describe how the method of sub-super solutions, together with the regularity result, which holds if the function $\varphi$ and the tensor field $h$ satisfy the additional conditions (0.1) (v)-(vi), allow to show that the $\lambda$-Liouville property holds for $C^{0} \cap W_{l o c}^{1, p}$ functions if and only if it holds for $C^{1}$ functions.

The first lemma clarifies the role of the ellipticity condition (0.1) (iv).

Lemma 1.1. Assume that condition (0.1) (iv) holds. Then, for every $\xi, \eta \in$ $T_{x} M$ we have

$$
h\left(|\xi|^{-1} \varphi(|\xi|) \xi-|\eta|^{-1} \varphi(\eta) \eta, \xi-\eta\right) \geq 0
$$

with equality if and only if $\xi=\eta$.

Proof. Let $X_{t}$ be the vector field in $T_{x} M$ defined by $X_{t}=t \eta+(1-t) \xi$, and set $\zeta=\xi-\eta$. Then the left hand side of (1.1) is equal to

$$
\begin{aligned}
h\left(\frac{\varphi\left(\left|X_{1}\right|\right)}{\left|X_{1}\right|} X_{1}, \zeta\right)-h\left(\frac{\varphi\left(\left|X_{0}\right|\right)}{\left|X_{0}\right|} X_{0}, \zeta\right)=\int_{0}^{1} \frac{d}{d t} h\left(\frac{\varphi\left(\left|X_{t}\right|\right)}{\left|X_{t}\right|} X_{t}, \zeta\right) d t \\
\quad=\int_{0}^{1}\left\{\frac{\varphi\left(\left|X_{t}\right|\right)}{\left|X_{t}\right|} h(\zeta, \zeta)+\left[\varphi^{\prime}\left(\left|X_{t}\right|\right)-\frac{\varphi\left(\left|X_{t}\right|\right)}{\left|X_{t}\right|}\right] \frac{\left\langle X_{t}, \zeta\right\rangle h\left(X_{t}, \zeta\right)}{\left|X_{t}\right|^{2}}\right\} d t
\end{aligned}
$$

and the required conclusion follows at once from (0.1) (iv). 
Lemma 1.2. Assume that $\varphi$ and $h$ satisfy conditions (0.1) (i)-(iv), and let $\Omega$ be a bounded open set. If $u, v \in C^{0}(\bar{\Omega}) \cap W_{\text {loc }}^{1, p}(\Omega)$ satisfy

$$
\begin{cases}\mathcal{L}_{\varphi, h} u \geq \mathcal{L}_{\varphi, h} v & \text { in } \Omega \\ u \leq v & \text { on } \partial \Omega\end{cases}
$$

then

$$
u \leq v \quad \text { in } \bar{\Omega} \text {. }
$$

Proof. Clearly, it suffices to prove that for every $\delta>0$, we have

$$
u \leq v+\delta \text { on } \Omega
$$

Toward this aim, fix $\delta>0$, and let $\tilde{\Omega}$ be an open set with smooth boundary such that

$$
\mathcal{O}=\{x \in \Omega: u(x)>v(x)+\delta\} \Subset \tilde{\Omega} \Subset \Omega .
$$

To construct $\tilde{\Omega}$ choose a smooth, non-negative function $\omega$ such that $\omega \equiv 1$ on $\mathcal{O}$ and $\equiv 0$ in $M \backslash \Omega$. If $c \in(1 / 4,3 / 4)$ is a regular value of $\omega$ (which exists by Sard's theorem) then we may set $\tilde{\Omega}=\{x: \omega>c\}$.

Let also $\alpha \in C^{1}(\mathbb{R})$ be such that $\alpha(t)=0$ if $t \leq \delta$, and $\alpha^{\prime}(t)>0$ if $t>\delta$ (so that $\alpha(t)>0$ there). Let $W$ be the vector field defined by

$$
W=\alpha(u-v)\left[\frac{\varphi(|\nabla u|)}{|\nabla u|} h(\nabla u, \cdot)^{\sharp}-\frac{\varphi(|\nabla v|)}{|\nabla v|} h(\nabla v, \cdot)^{\sharp}\right] .
$$

Note that, the definition of $\alpha$ and $\tilde{\Omega}$ imply that $W$ vanishes in a neighborhood of $\partial \tilde{\Omega}$ and since $u, v \in W_{l o c}^{1, p}(\Omega), W \in L^{1}(\tilde{\Omega})$. Further, a computation that uses (1.2) shows that

$$
\begin{aligned}
\operatorname{div} W= & \alpha(u-v)\left[\mathcal{L}_{\varphi, h} u-\mathcal{L}_{\varphi, h} v\right]+ \\
& \alpha^{\prime}(u-v) h\left(\frac{\varphi(|\nabla u|)}{|\nabla u|} \nabla u-\frac{\varphi(|\nabla v|)}{|\nabla v|} \nabla v, \nabla u-\nabla v\right) \\
\geq & \alpha^{\prime}(u-v) h\left(\frac{\varphi(|\nabla u|)}{|\nabla u|} \nabla u-\frac{\varphi(|\nabla v|)}{|\nabla v|} \nabla v, \nabla u-\nabla v\right) .
\end{aligned}
$$

Denote by $\rho$ the distance function from $\partial \tilde{\Omega}$, with the convention that $\rho(x)>0$ if $x \in \tilde{\Omega}$ and $\rho(x) \leq 0$ if $x \in M \backslash \tilde{\Omega}$, so that $\rho$ is the radial coordinate in the Fermi coordinates with respect to $\partial \tilde{\Omega}$, and, by Gauss Lemma, $|\nabla \rho|=1$. 
Let $\tilde{\Omega}_{\epsilon}=\{x \in \tilde{\Omega}: \rho(x)>\epsilon\}$, and let $\psi_{\epsilon}$ be the Lipschitz function defined by

$$
\psi_{\epsilon}(x)= \begin{cases}1 & \text { if } x \in \tilde{\Omega}_{\epsilon} \\ \frac{1}{\epsilon} \rho(x) & \text { if } x \in \tilde{\Omega} \backslash \tilde{\Omega}_{\epsilon} \\ 0 & \text { if } x \notin \tilde{\Omega} .\end{cases}
$$

Note that, since $W$ vanishes in a neighborhood of $\partial \tilde{\Omega}$, for every sufficiently small $\epsilon>0, W$ vanishes off $\tilde{\Omega}_{\epsilon}$, and by definition of weak divergence, we have

$$
\left\langle\operatorname{div} W, \psi_{\epsilon}\right\rangle=-\int\left\langle W, \nabla \psi_{\epsilon}\right\rangle=-\frac{1}{\epsilon} \int_{\tilde{\Omega} \backslash \tilde{\Omega}_{\epsilon}}\langle W, \nabla \rho\rangle=0
$$

whence, using (1.5), and letting $\epsilon \rightarrow 0$, we deduce that

$$
\int_{\tilde{\Omega}} \alpha^{\prime}(u-v) h\left(\frac{\varphi(|\nabla u|)}{|\nabla u|} \nabla u-\frac{\varphi(|\nabla v|)}{|\nabla v|} \nabla v, \nabla u-\nabla v\right) \leq 0 .
$$

According to Lemma 1.1, the expression involving $h$ is non-negative, and strictly positive if $\nabla u \neq \nabla v$. Since $\alpha^{\prime} \geq 0$, it follows that the integrand is a.e. equal to zero in $\tilde{\Omega}$.

To conclude, assume by contradiction that $\mathcal{O}$ is non-empty. Since $\alpha(u-v)>0$ in $\mathcal{O}$, again by Lemma 1.1, we conclude that $\nabla u=\nabla v$ a.e. in $\mathcal{O}$. But then, $u-v$ is constant in each connected component of $\mathcal{O}$ (see, e.g., [7, Lemma 1.16]). But since $u=v+\delta$ on $\partial \mathcal{O}$, this contradicts the definition of $\mathcal{O}$.

We note that we will apply our comparison principle to functions that are not necessarily $C^{1}(\Omega)$, most notably to radial functions on the underlying manifold, which are in general only Lipschitz. An alternative proof of the comparison principe valid for Lipschitz functions may be obtained by using Lemma 1.1 to adapt to the case of the operator $\mathcal{L}_{\varphi, h}$ the comparison principle for the $\varphi$-Laplacian contained in [18, Proposition 6.1] (see also [21, Lemma 3]).

We also remark that the conclusion of the lemma holds if we replace the assumption that $u, v \in C(\bar{\Omega})$ and $u \leq v$ on $\partial \Omega$, with the assumption

$$
\limsup _{x \rightarrow \partial \Omega} u(x) \leq \liminf _{x \rightarrow \partial \Omega} v(x) .
$$

Before stating the next result we recall that, given $q \in L_{l o c}^{1}(\Omega)$, a function $u \in C^{0}(M) \cap W_{l o c}^{1, p}(M)$ satisfies the inequality $\mathcal{L}_{\varphi, h} u \geq q(x) u$ in $\Omega$ in distributional sense if, for every nonnegative function $\psi \in C_{c}^{\infty}(\Omega)$

$$
-\int_{\Omega}|\nabla u|^{-1} \varphi(|\nabla u|) h(\nabla u, \nabla \psi) \geq \int_{\Omega} q(x) u \psi .
$$


Lemma 1.3. Assume that $\varphi$ and h satisfy conditions (0.1) (i)-(iv) and let $q(x) \in L_{\text {loc }}^{1}(\Omega)$. If $u_{i} \in L_{\text {loc }}^{\infty}(\Omega) \cap W_{\text {loc }}^{1, p}(\Omega), i=1, \ldots n$ are sub-solutions of $\mathcal{L}_{\varphi, h} u=q(x) u$, that is, they satisfy the differential inequality $\mathcal{L}_{\varphi, h} u \geq q(x) u$ in $\Omega$, then so does the function $\max \left\{u_{i}\right\}$. Similarly, if $v_{i}$ are super-solutions, then so is the function $\min \left\{v_{i}\right\}$.

The Lemma is proved in [14], noting that condition (0.1) (iv) implies assumption (H2) (see the discussion above), and that assumption (H3) is never used in the proof.

Classical results of Tolksdorf, [26], and Kura, [12], assert that the $L^{\infty}$ super- and sub-solution method can be applied to $p$-Laplace type operators in order to produce regular solutions to appropriate Dirichlet problems. As mentioned above, conditions (0.1) (i)-(vi) and (0.3) are precisely the assumptions needed to apply the Tolksdorf-Kura theory to operators in the form $\mathcal{L}_{\varphi, h}$

In the next lemma, which plays a central role in the paper, and will be extensively used in the sequel, we adapt the method of super- and subsolutions to establish an existence and $C^{1}$ regularity result for solutions of the equation $\mathcal{L}_{\varphi, h} u=q(x) u$ on $M$. The proof is modelled on that one gives in the case of the Laplace operator.

Lemma 1.4. Assume that $\varphi$ and h satisfy conditions (0.1) (i)-(vi) and (0.3), respectively. Let $q(x) \in L_{l o c}^{1}(M)$ and suppose that $u_{-}$and $u_{+} \in L_{l o c}^{\infty}(\Omega) \cap$ $W_{l o c}^{1, p}(\Omega)$ are a sub-respectively super-solution of the equation

$$
\mathcal{L}_{\varphi, h} u=q(x) u \quad \text { in } M .
$$

If $u_{-} \leq u_{+}$in $M$ then there exists a $C^{1}(M)$-solution $u$ of (1.7) satisfying $u_{-} \leq u \leq u_{+}$.

Proof. Let $\left\{\Omega_{n}\right\}$ be an exhaustion of $M$ by relatively compact domains with smooth boundary $\partial \Omega_{n}$, and for every $n$ consider the Dirichlet problem

$$
\begin{cases}\mathcal{L}_{\varphi, h} u=q(x) u & \text { in } \Omega_{n} \\ u=u_{-} & \text {on } \partial \Omega_{n} .\end{cases}
$$

Since $u_{-}, u_{+}$are bounded sub- and super-solutions of (1.8), by [12, Theorem 3.4], the problem has a solution $u_{n} \in\left(W^{1, p} \cap L^{\infty}\right)\left(\Omega_{n}\right)$ satisfying

$$
u_{-} \leq u_{n} \leq u_{+} \quad \text { on }, \Omega_{n},
$$

and, by [26, Theorem 1], $u_{n} \in C^{1, \alpha}\left(\Omega_{n}\right)$. Furthermore, again by [26] and by (1.9), the sequence $\left\{u_{n}\right\}_{n \geq 2}$ is $C^{1, \alpha}$-bounded on $\bar{\Omega}_{1}$, and since the embedding $C^{1, \alpha}\left(\bar{\Omega}_{1}\right) \hookrightarrow C^{1}\left(\bar{\Omega}_{1}\right)$ is compact, by the Ascoli-Arzela's Theorem, 
there exists a subsequence $\left\{u_{n, 1}\right\}$ which converges in $C^{1}\left(\bar{\Omega}_{1}\right)$ to a function $\tilde{u}_{1}$ satisfying

$$
\mathcal{L}_{\varphi, h} \tilde{u}_{1}=q(x) \tilde{u}_{1} \quad \text { and } \quad u_{-} \leq \tilde{u}_{1} \leq u_{+} \quad \text { in } \Omega_{1} .
$$

By induction, for every $k$ one finds a subsequence $\left\{u_{n, k}\right\}$ of $\left\{u_{n, k-1}\right\}$ such that $\left\{u_{n, k}\right\}$ converges in $C^{1}\left(\bar{\Omega}_{k}\right)$ to a function $\tilde{u}_{k}$ satisfying

$$
\tilde{u}_{k}=\tilde{u}_{k-1} \text { in } \bar{\Omega}_{k-1}, \quad \mathcal{L}_{\varphi, h} \tilde{u}_{k}=q(x) \tilde{u}_{k} \quad \text { and } \quad u_{-} \leq \tilde{u}_{k} \leq u_{+} \quad \text { in } \Omega_{k} .
$$

Setting $u=\tilde{u}_{k}$ on $\Omega_{k}$ gives the desired solution.

As a corollary of the above Lemma, we begin to prove, that if $\varphi$ and $h$ satisfy the all assumptions listed above, then the $\lambda$-Liouville property holds for $C^{1}$ functions if and only if it holds for $\left(C^{0} \cap W_{l o c}^{1, p}\right)(M)$ functions. This extends well known results for the Laplace operator, and allows one to define the $\mathcal{L}_{\varphi, h}$-parabolicity (resp. stochastic completeness) of a manifold using the degree of regularity appropriate for the situation at hand.

Lemma 1.5. Assume that $\varphi$ and $h$ satisfy the conditions listed in the statement of Lemma 1.4. Then operator $\mathcal{L}_{\varphi, h}$ has the $\lambda$-Liouville property for $C^{0}(M) \cap W_{l o c}^{1, p}(M)$ functions if and only if the property holds for $C^{1}(M)$ functions.

Proof. Only the sufficiency of the condition requires proof. We consider only the case where $\lambda=0$. The case $\lambda>0$ is similar. To this end, assume that $u \in C^{0}(M) \cap W_{l o c}^{1, p}(M)$ is not constant, bounded above and satisfies $\mathcal{L}_{\varphi, h} u \geq 0$

By suitably translating $u$ we may assume that the sets $\Omega_{+}=\{x: u(x)>0\}$ and $\Omega_{-}=\{x: u(x)<0\}$ are both not empty, and choose $q \in C_{c}^{\infty}(M)$ nonnegative, and non-identically zero, with support contained in $\Omega_{-}$. It follows from Lemma 1.3 that $u_{-}=\max \{u, 0\}$ is nonnegative, non-constant and satisfies $\mathcal{L}_{\varphi, h} u_{-} \geq q(x) u_{-}$on $\mathrm{M}$. On the other hand, if $u_{+}$is a constant larger than $\sup u$, then $u_{+}$solves $\mathcal{L}_{\varphi, h} u_{+} \leq q(x) u_{+}$and $u_{-} \leq u_{+}$. According to Lemma 1.4, the equation $\mathcal{L}_{\varphi, h} u=q(x) u$ has a solution $u \in C^{1}(M)$ satisfying $u_{-} \leq u \leq u_{+}$. In particular, $u$ is bounded above, non constant, nonnegative, and since $q$ is nonnegative, $u$ is $\mathcal{L}_{\varphi, h}$-subharmonic.

We therefore conclude that if the $\lambda$-Liouville property holds for $C^{1}$ functions, then a contradiction is reached, and the only bounded above, $C^{0}(M) \cap$ $W_{l o c}^{1, p}(M) \mathcal{L}_{\varphi, h}$-subharmonic functions are necessarily constant, i.e., the $\lambda$ Liouville property holds for $C^{0} \cap W_{l o c}^{1, p}$ functions.

The conclusion of the next Proposition holds assuming only that $\varphi$ and $h$ satisfy the restricted set of conditions (0.1) (i)-(iv). 
Proposition 1.6. Assume that $\varphi$ satisfies conditions (0.1) (i)-(iv). Then the following implications hold:

(a) If the $\lambda$-weak maximum principle for $C^{1}$ (resp. for $C^{0} \cap W_{l o c}^{1, p}$ ) functions holds, then so does the $\lambda$-Liouville property;

(b) If the $\lambda$-Khas'minskii test holds then so does the $\lambda$-weak maximum principle for $C^{1}$ functions.

Proof. To prove (a), assume that the $\lambda$-weak maximum principle holds on $M$ (for functions in the appropriate regularity classes).

If $\lambda=0$, let $u$ be bounded above and satisfy $\mathcal{L}_{\varphi, h} u \geq 0$, on $M$. If $u$ were nonconstant, this would contradict the 0 -weak maximum principle. Therefore $u$ is constant, showing that the 0-Liouville property for $C^{1}$ (resp. $\left.C^{0} \cap W_{l o c}^{1, p}\right)$ functions holds.

If $\lambda>0$, let $u$ be a nonnegative, bounded above function satisfying $\mathcal{L}_{\varphi, h} u \geq \lambda u$ on $M$. If $u$ is not identically zero, then, for every $0<\eta<$ $u^{*}=\sup u, u$ satisfies $\inf \mathcal{L}_{\varphi, h} u \geq \lambda \eta$ on the set $\Omega_{\eta}=\{x: u(x)>\eta\}$, contradicting the assumed validity of the $\lambda$-weak maximum principle (under the appropriate regularity conditions).

To prove (b), suppose that the $\lambda$-Khas'minskii test holds, so that there exists a compact set $K$ and for every $\epsilon>0$ and every $x_{o} \notin K$ there exists a positive $\lambda$-superharmonic function $\gamma$ on $M \backslash K$, which tends to infinity at infinity and is such that $\gamma\left(x_{o}\right)<\epsilon$.

Assume by contradiction that the $\lambda$-Liouville property for $C^{1}$ functions is not valid.

Thus, if $\lambda=0$, there exists a function $u \in C^{1}(M)$ which is non-constant, bounded above and satisfies $\inf _{\Omega_{\eta}} \mathcal{L}_{\varphi, h} u \geq 0$ for some $\eta<u^{*}$. Since $\varphi$ increasing and $h$ positive definite, according to [19], the strong maximum principle holds for $\mathcal{L}_{\varphi, h}$. It follows that $u$ cannot attain its maximum on $\Omega_{\eta}$, so that $\bar{\Omega}_{\eta}$ is not compact, and by choosing $\eta$ closer to $u^{*}$, if needed, we may assume that $K \cap \Omega_{\eta}=\emptyset$. Pick $x_{o} \in \Omega_{\eta}$ such that $u\left(x_{o}\right)>\eta+\left(u^{*}-\eta\right) / 2=\left(u^{*}+\eta\right) / 2$, and let $\gamma$ be a function with the properties listed above, and satisfying $\gamma\left(x_{o}\right)<\left(u^{*}-\eta\right) / 2$. Finally, let $A$ be the connected component containing $x_{o}$ of the set $\{x: u(x)>\eta+\gamma(x)\}$. Since $\gamma>0$ and tends to infinity at infinity, $\bar{A}$ is a compact subset of $\Omega_{\eta}$, so that $\mathcal{L}_{\varphi, h} u \geq 0 \geq \mathcal{L}_{\varphi, h}(\eta+\gamma)$ on $A$ and $u=\eta+\gamma$ on $\partial A$. Thus, by comparison, $u \leq \eta+\gamma$ on A, thus contradicting the definition of $A$.

The case where $\lambda>0$ is similar. Again, we assume that $u$ is bounded above, and that, for some $\eta<u^{*}$

$$
\mathcal{L}_{\varphi, h} u \geq c>0 \quad \text { on } \Omega_{\eta} .
$$

Then $u$ does not attain its maximum on the set $\Omega_{\eta}$, and it may be assumed that $\Omega_{\eta} \cap K=\emptyset$, and that $\lambda\left(u^{*}-\eta\right)<c$. 
We choose $x_{o} \in \Omega_{\eta}$ such that $u\left(x_{o}\right)>\left(u^{*}+\eta\right) / 2$, and a positive function $\gamma$ which satisfies $\mathcal{L}_{\varphi, h} \gamma \leq \lambda \gamma$ on $M \backslash K$ and $\gamma\left(x_{o}\right)<\left(u^{*}-\eta\right) / 2$. If $A$ is the connected component containing $x_{o}$ of the set $\{x: u(x)>\eta+\gamma(x)\}$, then $\bar{A}$ is compact in $\Omega_{\eta}$, and, by definition $\gamma<u^{*}-\eta$ in $A$, so that

$$
\mathcal{L}_{\varphi, h} u \geq c>\lambda\left(u^{*}-\eta\right) \geq \lambda \gamma \geq \mathcal{L}_{\varphi, h} \gamma=\mathcal{L}_{\varphi, h}(\eta+\gamma)
$$

in $A$, and a contradiction is reached as in the case $\lambda=0$.

We can now complete the proof of Theorem A.

Proof of Theorem A. It is trivial that (i) implies (ii). The implication (ii) $\Rightarrow$ (iii) is the content of Lemma 1.6 (a), and the implication (iii) $\Rightarrow$ (iv) follows from Lemma 1.5.

It remains to prove that (iv) implies (i). To this end, suppose first that $\lambda=0$, and let $u$ be a bounded above function in $C^{0}(M) \cap W_{l o c}^{1, p}(M)$. By adding to it a suitable constant we may assume that $u^{*}>0$. Assume by contradiction that there exists $0<\eta<u^{*}$ such that

$$
\mathcal{L}_{\varphi, h} u \geq 0 \text { on } \Omega_{\eta} \text {. }
$$

If $\eta^{\prime} \in\left(\eta, u^{*}\right)$, then the function defined by

$$
v=\max \left\{u, \eta^{\prime}\right\}
$$

is not constant, and, since both $u_{1}=u$ and the constant function $u_{2}=\eta$ are solutions of $\mathcal{L}_{\varphi, h} u \geq 0$ in $\Omega_{\eta} \backslash \Omega_{\eta^{\prime}}$, by Lemma 1.3, $v$ satisfies $\mathcal{L}_{\varphi, h} v \geq 0$ in $M$. But then (iv) implies that $v$ is constant, yielding the required contradiction.

The case where $\lambda>0$ is similar. Again, we assume by contradiction that there exists $\eta<u^{*}$ such that

$$
\mathcal{L}_{\varphi, h} u \geq c>0 \quad \text { on } \Omega_{\eta} .
$$

By adding a constant to $u$, we may assume that $\eta<0<u^{*}$ and that $\lambda u^{*}<c$, so that $u$ satisfies

$$
\mathcal{L}_{\varphi, h} u \geq c>\lambda u^{*} \geq \lambda u \quad \text { on } \Omega_{\eta} .
$$

Since, clearly $u_{0} \equiv 0$ satisfies

$$
\mathcal{L}_{\varphi, h} u_{0}=\lambda u_{0} \quad \text { on } M
$$

by Lemma 1.3 , the function

$$
v=u_{+}= \begin{cases}\max \left\{u, u_{0}\right\} & \text { on } \Omega_{\eta} \\ u_{0} & \text { on } M \backslash \Omega_{0},\end{cases}
$$

is a $C^{0}(M) \cap W_{l o c}^{1, p}(M)$ non-identically vanishing solution of $\mathcal{L}_{\varphi, h} v \geq \lambda v$ satisfying $0 \leq v \leq u^{*}$. The required contradiction now follows from (iv). The last assertion in the statement is the content of Proposition 1.6 (b). 
Corollary 1.7. Under the hypotheses of Theorem A, if the $\lambda$-Liouville property holds for some $\lambda_{o}>0$, then it holds for every $\lambda>0$.

\section{Model manifolds, and applications}

In this section, we consider the case where the underlying manifolds is a model in the sense of Greene and Wu. As it is often the case, some one sided implications become equivalences in this simplified setting, but still the results obtained may help to shed light on the situation in the general case.

We begin by considering the case of the $\varphi$-Laplacian. In this case we obtain a complete characterization of the validity of the $\lambda$-Liouville property in terms of volume growth. Using this, we then prove the comparison result which is the content of Theorem B. It should be pointed out that, although the result on models is expressed in terms of volume growth, the result for general manifolds (which follows from the aforementioned comparison) depends on curvature assumptions.

So let $\sigma$ be a smooth function with the properties listed before the statement of Theorem B, and let $\left(\tilde{M}, \widetilde{\langle,\rangle_{\sigma}}\right)$ be the $m$-dimensional model manifold defined by $\sigma$.

Lemma 2.1. Let $\sigma$ be a $C^{1}$ positive function defined on $(0,+\infty)$ and assume that the function $\mathcal{V}_{\lambda, c}$ defined by

$$
\mathcal{V}_{\lambda, c}(r)= \begin{cases}\varphi^{-1}\left(c \sigma(r)^{1-m}\right) & \text { if } \lambda=0 \\ \varphi^{-1}\left(c \sigma(r)^{1-m} \int_{R}^{r} \sigma(t)^{m-1} d t\right) & \text { if } \lambda>0,\end{cases}
$$

is defined on $[R,+\infty)$ for some $R>0$, and every $c>0$ sufficiently small. Then the function

$$
\alpha(r)=\alpha_{\lambda, c}(r)=\int_{R}^{r} \mathcal{V}_{\lambda, c}(t) d t+\alpha_{o}
$$

is defined in $[R,+\infty)$ and there satisfies

$$
\left[\sigma^{m-1} \varphi\left(\alpha^{\prime}\right)\right]^{\prime}= \begin{cases}0 & \text { if } \lambda=0 \\ c \sigma^{m-1} & \text { if } \lambda>0 .\end{cases}
$$

Moreover, given $\lambda \geq 0, \epsilon>0$ and $r_{o}>R$, for $c, \alpha_{o}>0$ sufficiently small we have

$$
\begin{aligned}
& \text { (i) } \alpha\left(r_{o}\right)<\epsilon \\
& \text { (ii) } \sigma^{1-m}\left[\sigma^{m-1} \varphi\left(\alpha^{\prime}\right)\right]^{\prime} \leq \lambda \alpha \quad \text { in }[R,+\infty) \text {. }
\end{aligned}
$$


Clearly, the assumption on $\mathcal{V}_{\lambda, c}$ is automatically satisfied if $\varphi(t) \rightarrow+\infty$ as $t \rightarrow+\infty$.

Proof. The first assertion follows from a straightforward computation. The second assertion is trivial if $\lambda=0$, so assume that $\lambda>0$. Choosing $\alpha_{o}=c / \lambda$, it follows from (2.3) that

$$
\sigma^{1-m}\left[\sigma^{m-1} \varphi\left(\alpha^{\prime}\right)\right]^{\prime}=c \leq \lambda \alpha_{o} \leq \lambda \alpha \quad \text { on }[R,+\infty),
$$

and then the inequality $\alpha\left(r_{o}\right)<\epsilon$ holds provided $c>0$ is sufficiently small.

Proposition 2.2. Let $(\tilde{M}, \widetilde{\langle,\rangle})$ be an m-dimensional model manifold. Assume that the function $\varphi$ satisfies conditions (0.1) (i)-(iv), and that the function $\mathcal{V}_{\lambda, c}$ defined in (2.1) is defined on $[R,+\infty)$ for some $R>0$ and every sufficiently small $c>0$. Then the following properties of the $\varphi$-Laplacian $\mathcal{L}_{\varphi}$ on $\tilde{M}$ are equivalent:

(a) The $\lambda$-Khas'minskii test holds on $\tilde{M}$;

(b) The $\lambda$-weak maximum principle for $C^{1}$ functions holds on $\tilde{M}$;

(c) The function $\mathcal{V}_{\lambda, c} \notin L^{1}(+\infty)$ for every sufficiently small c.

Remark 2.3. The proposition shows that if the $\lambda$-Khas'minskii test is valid for some $\lambda_{o}>0$ then it is valid for every $\lambda>0$.

Recalling that on a model the volume of the sphere and the ball centered at 0 of radius $r$ are given by $\operatorname{vol} \partial B_{r}=c_{m} \sigma(r)^{m-1}$ and $\operatorname{vol} B_{r}=$ $c_{m} \int_{0}^{r} \sigma(t)^{m-1} d t$, the condition in (c) above may by be expressed in more geometrical terms by saying that

$$
\begin{aligned}
& \varphi^{-1}\left(c \frac{1}{\operatorname{vol} \partial B_{r}}\right) \notin L^{1}(+\infty) \text { if } \lambda=0, \\
& \varphi^{-1}\left(c \frac{\operatorname{vol} B_{r}}{\operatorname{vol} \partial B_{r}}\right) \notin L^{1}(+\infty) \text { if } \lambda>0 \text {. }
\end{aligned}
$$

In the case of the $p$-Laplacian, the condition in turn reads

$$
\begin{aligned}
& \left(\frac{1}{\operatorname{vol} \partial B_{r}}\right)^{1 /(p-1)} \notin L^{1}(+\infty) \text { if } \lambda=0, \\
& \left(\frac{\operatorname{vol} B_{r}}{\operatorname{vol} \partial B_{r}}\right)^{1 /(p-1)} \notin L^{1}(+\infty) \quad \text { if } \lambda>0,
\end{aligned}
$$

which, for the standard Laplace operator $(p=2)$ reduce to the well known necessary and sufficient condition for the parabolicity, respectively stochastic completeness, of models. 
Observe also that if we assume that $\varphi$ satisfies the structural condition (0.1) (iii) with $\kappa=0$ then condition (c) is implied by (2.6). It was proved in [23], by entirely different methods, that, under the stated assumption on $\varphi$, the condition for $\lambda=0$ implies the $\varphi$-parabolicity of arbitrary manifolds. This can be recovered using the comparison result contained in Theorem B (i). However, the assumption (0.9), on which the comparison rests, expresses a condition on the radial Ricci curvature of the manifold.

Proof of Proposition 2.2. According to Proposition 1.6, the implication (a) $\Rightarrow$ (b) holds on a general manifold.

To prove that $(\mathrm{b}) \Rightarrow(\mathrm{c})$, let $R$ be as in the statement of Lemma 2.1, and let $\alpha$ be the function defined in (2.2). Define a function $v \in C^{1}(M)$ by setting it equal to $\alpha(r(x))$ in $M \backslash B_{R}$ and extending to $M$ is such a way that $v(x)<\alpha_{o}$ in $B_{R}$. Since $\nabla v=\alpha^{\prime} \nabla r, \alpha^{\prime}>0$, and $\Delta r(x)=(m-1) \sigma^{\prime} / \sigma$,

$$
\mathcal{L}_{\varphi} v(x)=\operatorname{div}\left(\varphi\left(\alpha^{\prime}\right) \nabla r\right)(x)=\sigma^{1-m}(r(x))\left[\sigma^{m-1} \varphi\left(\alpha^{\prime}\right)\right]^{\prime}(r(x))
$$

on the set $\left\{x: v(x)>\alpha_{o}\right\}=M \backslash B_{R}$. Thus, applying (2.3) we deduce that

$$
\mathcal{L}_{\varphi} v(x)= \begin{cases}0 & \text { if } \lambda=0 \\ c & \text { if } \lambda>0\end{cases}
$$

so that, if (b) holds, then $v$ cannot be bounded above. Therefore $\alpha(r)$ diverges as $r \rightarrow+\infty$, i.e., $\mathcal{V}_{\lambda, c} \notin L^{1}(+\infty)$.

Finally, to prove that (c) $\Rightarrow$ (a), let again $R$ be as in Lemma 2.1. Given $x_{o} \notin B_{R}$, and $\epsilon>0$, let $\alpha$ and $v$ be defined as above, with $c$ and $\alpha_{o}$ small enough as to guarantee that $\alpha\left(r\left(x_{0}\right)\right)<\epsilon$ and that (2.4) (ii) holds on $[R,+\infty)$. Then $v \in C^{1}(M)$ satisfies $v\left(x_{o}\right)<\epsilon$, and, by (c), $v(x) \rightarrow+\infty$ as $r(x) \rightarrow+\infty$. Moreover, by (2.4) and the computation done in (2.7),

$$
\mathcal{L}_{\varphi} v(x)=\sigma^{1-m}(r(x))\left[\sigma^{m-1} \varphi\left(\alpha^{\prime}\right)\right]^{\prime}(r(x)) \leq \lambda \alpha(r(x))=\lambda v(x)
$$

on $M \backslash B_{R}$, showing that the $\lambda$-Khas'minskii test holds.

Applying Proposition 2.2 we can now give a proof of Theorem B. For the case of the Laplacian see [5, pp. 221-222].

Proof of Theorem B. To prove (i), let $R>0$ and fix $x_{o} \in M \backslash B_{R}(o)$ and $\epsilon>0$. Let $\alpha$ be the function considered in Proposition 2.2, and let $v(x)=\alpha(r(x))$. Arguing as in the proof of Proposition 2.2, we see that for every $c>0$ sufficiently small we have $v\left(x_{o}\right)<\epsilon$ and the differential inequality

$$
\mathcal{L}_{\varphi} v(x)=\left[\varphi\left(\alpha^{\prime}\right)\right]^{\prime}+\varphi\left(\alpha^{\prime}\right) \Delta r(x) \leq\left[\varphi\left(\alpha^{\prime}\right)\right]^{\prime}+(m-1) \frac{\sigma^{\prime}}{\sigma} \varphi\left(\alpha^{\prime}\right)=\lambda v
$$

holds on $M \backslash\left[B_{R}(o) \cup \operatorname{cut}(o)\right]$. 
Since $v$ is locally Lipschitz, and $\alpha^{\prime}>0$, by an extension of an argument of Yau's (see [28]) the inequality holds weakly in $M \backslash B_{R}(o)$. On the other hand, since the weak maximum principle holds on $\tilde{M}$, by the characterization given in Proposition 2.2, for every sufficiently small $c>0$ we have $\mathcal{V}_{\lambda, c} \notin L^{1}(+\infty)$ and therefore, for all such $c>0, v(x) \rightarrow+\infty$ as $x \rightarrow \infty$. Thus the $\lambda$-Khas'minskii test holds on $M$ and the conclusion follows from Proposition 1.6.

To prove (ii), let $\alpha$ be the function considered above, let $v \in C^{1}(M)$ be equal to $\alpha(r(x))$ on $M \backslash B_{R}(o)$ and less than $\alpha_{o}$ in $B_{R}(o)$, and let $\tilde{v} \in C^{1}(\tilde{M})$ be defined in a similar way. Since $\Delta r \geq(m-1) \sigma^{\prime} / \sigma=\tilde{\Delta} \tilde{r}$, the computation that leads to (2.8), and the the properties of $\alpha$ yield the inequality

$$
\mathcal{L}_{\varphi} v(x) \geq\left[\varphi\left(\alpha^{\prime}\right)\right]^{\prime}+(m-1) \frac{\sigma^{\prime}}{\sigma} \varphi\left(\alpha^{\prime}\right)= \begin{cases}0 & \text { if } \lambda=0 \\ c & \text { if } \lambda>0\end{cases}
$$

in $M \backslash B_{R}(o)=\left\{x: v(x)>\alpha_{o}\right\}$. On the other hand, if the $\lambda$-weak maximum principle didn't hold on $\tilde{M}$, the function $\tilde{v}$ would be bounded above, by Proposition 2.2, and therefore so would be $v$. But then, (2.9) would contradict the assumed validity of the $\lambda$-weak maximum principle on $M$.

Corollary 2.4. Let $(M,\langle\rangle$,$) be a complete Riemannian manifold, and as-$ sume that the differential inequality

$$
\Delta r \geq(m-1) \frac{\sigma^{\prime}}{\sigma}
$$

holds in the complement of the cut locus. If, for every $c>0$ sufficiently small,

$$
\varphi^{-1}\left(\frac{c}{\sigma(r)^{m-1}}\right) \notin L^{1}(+\infty), \quad \text { or } \quad \varphi^{-1}\left(c \frac{\int_{0}^{r} \sigma(t)^{m-1} d t}{\sigma(r)^{m-1}}\right) \notin L^{1}(+\infty)
$$

then the 0-weak, or the $\lambda$-weak maximum principle, respectively, hold on $M$.

Remark 2.5. As mentioned above, both (0.9) and its counterpart (0.10), which are the main geometrical assumptions in Theorem B, are implied by suitable curvature bounds. For instance, if we assume that the radial Ricci curvature satisfies

$$
\operatorname{Ric}_{M}(\nabla r, \nabla r) \geq-(m-1) G(r)
$$

where $G \in C^{\infty}([0,+\infty))$ is even at the origin, and satisfies

$$
\inf _{r>0} \frac{G^{\prime}(r)}{G(r)^{3 / 2}}>-\infty
$$


then the inequality (0.9) holds with

$$
\sigma(r)=\exp \left(A \int_{0}^{r} G(s)^{1 / 2} d s\right)-1
$$

provided $A>0$ is sufficiently large (see, e.g., [22]). The inequality (0.9) in turns implies that $\operatorname{vol} \partial B_{r}(o) / \sigma(r)^{m-1}$ is a decreasing function of $r$. In particular, $\operatorname{vol} \partial B_{r}(o) \leq c_{m} \sigma(r)^{m-1}$, and, by an argument of J. Cheeger, M. Gromov and M. Taylor, [1], the function

$$
\operatorname{vol} B_{r}(o) / \int_{0}^{r} \sigma(t)^{m-1} d t
$$

is also decreasing. By taking derivatives, we deduce that

$$
\frac{\operatorname{vol} B_{r}}{\operatorname{vol} \partial B_{r}} \geq \frac{\int_{0}^{r} \sigma(t)^{m-1} d t}{\sigma(r)^{m-1}}
$$

Thus, (2.10) implies that, for every $c>0$ small enough,

$$
\varphi^{-1}\left(\frac{c}{\operatorname{vol} \partial B_{r}(o)}\right) \notin L^{1}(+\infty), \quad \text { or } \quad \varphi^{-1}\left(c \frac{\operatorname{vol} B_{r}(o)}{\operatorname{vol} \partial B_{r}(o)}\right) \notin L^{1}(+\infty),
$$

respectively, and we see that the curvature condition (2.11) implies the volume growth condition (2.5) which was seen to characterize the validity of the $\lambda$-weak maximum principle on models.

Remark 2.6. According to Theorem A, if assumptions (0.1) (i)-(vi) and (0.3) hold, then, the validity of the 0-Khas'minskii test implies that the the operator $\mathcal{L}_{\varphi, h}$ satisfies the 0-Liouville property, that is that the manifold $M$ is $\mathcal{L}_{\varphi, h}$-parabolic. Proposition 2.2 shows that in the case of the $\varphi$-Laplacian on model manifolds, the validity of the 0 -Khasminskii test and the $\mathcal{L}_{\varphi^{-}}$ parabolicity of the manifold are equivalent properties.

A theorem of Z. Kuramochi for Riemann surfaces, [13], whose proof was simplified and extended to arbitrary dimensions by M. Nakai, see [16] and [24], asserts that for the Laplace operator this equivalence holds on arbitrary Riemannian manifold.

The proof is based on a capacity approach, and on Green's functions techniques, and as such is unlikely to carry over to the case of more general operators like the $\varphi$-Laplacian, or the operator $\mathcal{L}_{\varphi, h}$. We are indebted to I. Holopainen who pointed out to us that, in the case of the $p$-Laplacian, or more generally the $\mathcal{A}$-Laplacian, where both the capacity and Green's function techniques are available, the equivalence can be proved under the (rather stringent) assumptions that the operator satisfies a global Harnack inequality and that the manifold has only finitely many ends, and satisfies the volume doubling property. 
We outline here the argument, restricting ourselves to the case of the $p$-Laplacian for simplicity. By considering the complement of a compact set $K$ large enough the ends relative to $K$ are pairwise disjoint, we may further assume that the manifold has only one end. Assume therefore that the manifold $M$ is $p$-parabolic. In this context this means equivalently the validity of the 0 -Liouville property for the $p$-Laplacian, or the vanishing of the $p$-capacity of the ideal boundary of the manifold.

Arguing as in the proof of Lemma 2.15 in [10] we construct a $p$-harmonic function $u: M \backslash K \rightarrow R$ with the property that, for every $t \gg 1$

$$
\sup _{\partial B_{t}(o)} u(x) \geq C\left(\operatorname{cap}_{p}\left(\partial K, \partial B_{t}(o), M \backslash K\right)\right)^{1 /(1-p)},
$$

for some constant $C>0$ independent of $t$. Since the manifold satisfies the doubling condition, there exists $N$ such that $\partial B_{t}(o)$ is covered by at most $N$ balls of radius $t / 2$. On the other hand, since a global Harnack inequality holds for the $p$-Laplacian, there exists a constant $L$ independent of $r$ such that, if $v$ is positive and $p$-harmonic in $B_{2 r}(x)$ then

$$
\sup _{B_{r}(x)} v \leq L \inf _{B_{r}(x)} v .
$$

We conclude that there exists a positive constant $C_{1}$ such that, for every $t \gg 1$,

$$
\inf _{\partial B_{t}(o)} u(x) \geq C_{1}\left(\operatorname{cap}_{p}\left(\partial K, \partial B_{t}(o), M \backslash K\right)\right)^{1 /(1-p)} .
$$

The required conclusion that $u$ tends to $+\infty$ at infinity now follows since the assumption that the capacity of the ideal boundary is zero amounts to saying that the capacity on the right hand side tends to zero as $t \rightarrow+\infty$.

It seems to be an interesting problem to explore if the equivalence between the appropriate version of parabolicity and the validity of the corresponding form of the 0-Khas'minskii test holds in more complicated geometrical situation, or for the more general operators $\mathcal{L}_{\varphi}$ or $\mathcal{L}_{\varphi, h}$, where the homogeneity conditition (0.4) is not assumed.

Our next task is to extend to the operator $\mathcal{L}_{\varphi, h}$ on a manifold with a pole the sufficient condition for the validity of the $\lambda$-weak maximum principle given in Corollary 2.4. In accordance with the notation introduced, we let $o$ be a fixed reference point in $M$ and denote by $r(x)$ the distance function from $o$. We begin by recalling the expression of $\mathcal{L}_{\varphi, h} u$ when $u$ is a function depending only upon $r(x)$.

Lemma 2.7. Let $\alpha$ be a strictly monotonic $C^{2}$ function on $[R,+\infty)$ and set $v(x)=\alpha(r(x))$. Then, on $M \backslash\left(B_{R} \cup\right.$ cut $\left.(o)\right)$, we have

$$
\begin{aligned}
\mathcal{L}_{\varphi, h} v(x)=\left(\operatorname{sgn} \alpha^{\prime}\right)\left\{\left[\varphi\left(\left|\alpha^{\prime}\right|\right)\right]^{\prime} h(\nabla r, \nabla r)\right. & \\
& \left.+\varphi\left(\left|\alpha^{\prime}\right|\right)\left[\operatorname{div} h(\nabla r)+\langle h, \operatorname{Hess} r\rangle_{S^{2} T^{*} M}\right]\right\}
\end{aligned}
$$


Proof. Recalling the definition of $\mathcal{L}_{\varphi, h}$, we compute

$$
\begin{aligned}
\mathcal{L}_{\varphi, h} v(x)=\left(\operatorname{sgn} \alpha^{\prime}\right) \varphi\left(\left|\alpha^{\prime}\right|\right) \operatorname{div}\left[h(\nabla r, \cdot)^{\sharp}\right] & \\
& +\left\langle\nabla\left[\left(\operatorname{sgn} \alpha^{\prime}\right) \varphi\left(\left|\alpha^{\prime}\right|\right)\right], h(\nabla r, \cdot)^{\sharp}\right\rangle .
\end{aligned}
$$

Now,

$$
\left\langle\nabla\left[\left(\operatorname{sgn} \alpha^{\prime}\right) \varphi\left(\left|\alpha^{\prime}\right|\right)\right], h(\nabla r, \cdot)^{\sharp}\right\rangle=\left(\operatorname{sgn} \alpha^{\prime}\right)\left[\varphi\left(\left|\alpha^{\prime}\right|\right)\right]^{\prime} h(\nabla r, \nabla r) .
$$

On the other hand, if $E_{i}$ is a local orthonormal frame, denoting by $D_{E_{i}} Y$ the covariant derivative of the vector field $Y$ in the direction of $E_{i}$, we have

$$
\begin{aligned}
\operatorname{div}\left[h(\nabla r, \cdot)^{\sharp}\right] & =\sum_{i}\left\langle D_{E_{i}} h(\nabla r, \cdot)^{\sharp}, E_{i}\right\rangle \\
& =\sum_{i} E_{i} h\left(\nabla r, E_{i}\right)-h\left(\nabla r, D_{E_{i}} E_{i}\right) \\
& =\sum_{i}\left(D_{E_{i}} h\right)\left(\nabla r, E_{i}\right)+h\left(D_{E_{i}} \nabla r, E_{i}\right) \\
& =(\operatorname{div} h)(\nabla r)+\sum_{i, j} h\left(E_{i}, E_{j}\right) \operatorname{Hess} r\left(E_{i}, E_{j}\right) \\
& =(\operatorname{div} h)(\nabla r)+\langle h, \operatorname{Hess} r\rangle_{S^{2} T^{*} M},
\end{aligned}
$$

whence the required conclusion follows upon inserting the above identities into (2.13).

Assume now that $\alpha^{\prime}>0$, that $h$ satisfies

$$
h_{-}(r(x)) \leq h \leq h_{+}(r(x)), \quad \text { and } \quad|\operatorname{div} h|(x) \leq \beta(r(x)),
$$

for some positive functions $\beta$ and $h_{ \pm}:[0,+\infty) \rightarrow(0,+\infty)$ and that

$$
\text { Hess } r \leq \frac{\sigma^{\prime}}{\sigma}(\langle,\rangle-d r \otimes d r)
$$

where $\sigma$ is a smooth function with $\sigma^{\prime} \geq 0$. Then, choosing an orthonormal basis of $(\nabla r)^{\perp}$ which diagonalizes Hess $r$ (which is possible since $\operatorname{Hess} r(E, \nabla r)=0$ for every $E)$, we have

$$
h(\nabla r, \nabla r) \geq h_{-} \text {and }\langle h, \operatorname{Hess} r\rangle_{S^{2} T^{*} M} \leq(m-1) h_{+} \frac{\sigma^{\prime}}{\sigma} .
$$

Inserting in (2.12) it follows that

$$
\begin{aligned}
\mathcal{L}_{\varphi, h} v(x) & \leq h(\nabla r, \nabla r)\left\{\left[\varphi\left(\alpha^{\prime}\right)\right]^{\prime}+\frac{1}{h_{-}} \varphi\left(\alpha^{\prime}\right)\left[\beta(r)+(m-1) h_{+} \frac{\sigma^{\prime}}{\sigma}\right]\right\} \\
& =h(\nabla r, \nabla r)\left\{\left[\varphi\left(\alpha^{\prime}\right)\right]^{\prime}+\varphi\left(\alpha^{\prime}\right)(m-1) \frac{\tilde{\sigma}^{\prime}}{\tilde{\sigma}}\right\},
\end{aligned}
$$


where $\tilde{\sigma}$ is the function defined by

$$
(m-1) \frac{\tilde{\sigma}^{\prime}}{\tilde{\sigma}}=\frac{1}{h_{-}}\left[\beta(r)+(m-1) h_{+} \frac{\sigma^{\prime}}{\sigma}\right]
$$

Let $\tilde{\mathcal{V}}_{\lambda, c}$ be the function defined as in (2.1) using $\tilde{\sigma}$ instead of $\sigma$, namely,

$$
\tilde{\mathcal{V}}_{\lambda, c}(r)= \begin{cases}\varphi^{-1}\left(c \tilde{\sigma}(r)^{1-m}\right) & \text { if } \lambda=0 \\ \varphi^{-1}\left(c \tilde{\sigma}(r)^{1-m} \int_{R}^{r} \tilde{\sigma}(t)^{m-1} d t\right) & \text { if } \lambda>0\end{cases}
$$

We assume that, for some $R>0$ and every $c>0$ sufficiently small, the function $\tilde{\mathcal{V}}_{\lambda, c}$ is is defined on $[R,+\infty)$. Then the function

$$
\alpha(r)=\alpha_{\lambda, c}(r)=\int_{R}^{r} \tilde{\mathcal{V}}_{\lambda, c}(t) d t+\tilde{\alpha}_{o}
$$

is defined in $[R,+\infty)$ and satisfies the conclusion of Lemma 2.1.

We choose $\alpha_{o}=\lambda / c, c>0$ small enough that (2.4) holds. Inserting into (2.14) we obtain that

$$
\mathcal{L}_{\varphi, h} v(x) \leq \lambda v(x)
$$

pointwise in $M \backslash\left(B_{R}(o) \cup\right.$ cut $\left.(o)\right)$. In particular, if $o$ is a pole, the inequality holds in $M \backslash B_{R}(o)$.

We therefore state the following version of Corollary 2.4 .

Corollary 2.8. Let $M$ be a manifold with a pole. Notation and the assumptions being as in the preceding discussion, assume that the function $\tilde{\mathcal{V}}_{\lambda, c}$ is defined on $[R,+\infty)$ for some $R>0$ and all sufficiently small $c>0$, and that

$$
\tilde{\mathcal{V}}_{\lambda, c} \notin L^{1}(+\infty)
$$

Then the $\lambda$-Khas'minskii test for the operator $\mathcal{L}_{\varphi, h}$ is valid on $M$, and therefore so is the $\lambda$-weak maximum principle.

We end the paper with a discussion of a version of the Liouville property for functions belonging to suitable integrability classes. It is a classical result that if $(M,\langle\rangle$,$) is a (not necessarily complete) Riemannian manifold, then$ the following properties are equivalent:

(1) If $u \in C^{2}(M)$ (or, equivalently, $\left.u \in C^{0}(M) \cap W_{\text {loc }}^{1,2}(M)\right)$ is nonnegative, $\Delta u \leq 0$ (in distributional sense) and $u \in L^{1}(M)$ then $u \equiv 0$; 
(2) If $g(x)=g(x, o)$ is the Green kernel with singularity at $o \in M$, that is the minimal positive fundamental solution of $\Delta$ then either $g \equiv+\infty$ (that is, $M$ is parabolic) or $g<+\infty$ on $M \backslash\{o\}$ and

$$
\int_{M \backslash B_{1}(o)} g(x) d x=+\infty .
$$

Indeed, the implication (1) $\Rightarrow(2)$ follows from the fact that, if $g<+\infty$ on $M \backslash\{o\}$, then the function $u(x)=\min \{c, g(x)\}$ belongs to $C^{0}(M) \cap W_{l o c}^{1, p}(M)$, it is non-negative, non-identically zero, and satisfies $\Delta u \leq 0$. Thus, if (2.20) did not hold, $u$ would be in $L^{1}(M)$, and this would contradict (1).

The reverse implication is proved in [6], and we outline the argument for the convenience of the reader. If $g \equiv+\infty$ then $M$ is parabolic, and (1) holds without any integrability condition. So assume that $g<+\infty$ on $M \backslash\{o\}$, and that its integral over $M \backslash B_{1}(o)$ diverges. Assume by contradiction that there exists a non-negative, non-identically zero function $u$ which is superharmonic and integrable on $M$. By the maximum principle, $u$ is in fact strictly positive on $M$, and there exists $c>0$ s.t. $u>c$ on $B_{1}(o)$, and therefore for every sufficiently small $\epsilon>0, u \geq \epsilon g$ on $\partial B_{1}(o)$. Next let $B_{1}(o) \Subset \Omega_{1} \Subset \Omega_{2} \ldots \Omega_{n} \nearrow M$ be a sequence of relatively compact domains with smooth boundary, and let $g_{n}$ be the Dirichlet Green kernel of $\Omega_{n}$ with pole at $o$ so that $g_{n} \nearrow g$ on $M \backslash\{o\}$. Since $u \geq \epsilon g \geq \epsilon g_{n}$ on $\partial B_{1}(o)$ and $u>\epsilon g_{n}=0$ on $\partial \Omega_{n}$, by the comparison principle $u \geq \epsilon g_{n}$ on $\Omega_{n} \backslash B_{1}(o)$, whence, letting $n \rightarrow+\infty$, we deduce that $u \geq \epsilon g$ on $M \backslash B_{1}(o)$. Thus,

$$
\epsilon \int_{M \backslash B_{1}(o)} g(x) d x \leq \int_{M \backslash B_{1}(o)} u(x) d x<+\infty,
$$

which contradicts (2.20).

Assume now that $M$ is stochastically complete, and let $h_{t}(x, y)$ be its heat kernel, so that $\int_{M} h_{t}(x, y) d x=1$ for every $t>0$ and every $y \in M$. Furthermore, if $M$ is not parabolic, then $g(x, o)=\int_{0}^{+\infty} h_{t}(x, o) d t$. Thus

$$
\int_{M} g(x, o) d x=\int_{0}^{+\infty} \int_{M} h_{t}(x, o) d x=+\infty,
$$

and since $g \in L^{1}\left(B_{1}(o)\right.$ ) (indeed the local singularity is of the order of $r(x)^{2-n}$ ) we conclude that (2.20) holds.

In the case of models, the Green's function with singularity at the pole $o$ is given by

$$
g(x)=c_{m} \int_{r(x)}^{+\infty} \sigma(t)^{1-m} d t
$$


Indeed, a straightforward computation shows that $\Delta g=0$ in $M \backslash\{o\}$, and since $\sigma(t) \sim t$ as $t \rightarrow 0$, we have $g(x) \asymp r(x)^{m-2}$ as $r(x) \rightarrow 0$, as required. An application of Fubini's theorem shows that

$$
\begin{aligned}
\int_{M \backslash B_{1}(o)} g(x) d x & =\int_{1}^{+\infty} d r \sigma(r)^{m-1} \int_{r}^{+\infty} \frac{1}{\sigma(t)^{m-1}} d t \\
& =\int_{1}^{+\infty} d t \frac{1}{\sigma(t)^{m-1}} \int_{1}^{t} \sigma(r)^{m-1} d r
\end{aligned}
$$

and we conclude that (2.20) above holds if and only if

$$
\frac{1}{\sigma(t)^{m-1}} \int_{1}^{t} \sigma(r)^{m-1} \asymp \frac{\operatorname{vol} B_{t}}{\operatorname{vol} \partial B_{t}} \notin L^{1}(+\infty),
$$

which is the necessary and sufficient condition for the stochastic completeness of the model manifold $M$.

In [10] Holopainen considered similar kinds of Liouville type results for the $\mathcal{A}$-Laplacian. In particular he proved that if $u \in L^{q}(M)$ is a non-negative $p$-superhamonic (respectively $p$-subharmonic) function and $q<p-1$ (respectively, $q>p-1$ ), then $u \equiv 0$. He also showed that the conclusion fails at the critical exponent $q=p-1$, and posed the question of finding conditions similar to (2.22) which ensure that $L^{p-1}$ non-negative $p$-superharmonic functions vanish identically. In the sequel we will refer to this as the $L^{p-1}$-Liouville property.

The fact that the $L^{1}$-Liouville property is implied by the stochastic completeness on the manifold, and it is actually equivalent to it on models, suggests that $L^{p-1}$-Liouville property may be related to the $p$-stochastic completeness of the manifold, that is, to the validity of the $\lambda$-weak maximum principle, $\lambda>0$ for the $p$-Laplacian.

We are going to investigate this relationship in the case of models. Adapting the argument used in the linear case, one verifies that, having denoted by $g_{p}(x)$ a Green function for the $p$-Laplacian with pole at $o$ (for the construction of $g_{p}$, see, e.g., [8]), the $L^{p-1}$-Liouville property is equivalent to the fact that the following version of condition (2.20) holds:

$$
\int_{M \backslash B_{1}(o)} g_{p}(x)^{p-1} d x=+\infty .
$$

In the case of models, an explicit computation, similar to that performed in the linear case shows that if the function defined by

$$
g_{p}(x)=\int_{r(x)}^{+\infty} \sigma(t)^{-\frac{m-1}{p-1}} d t
$$


is not identically equal to $+\infty$, then it satisfies

$$
\Delta_{p} g_{p}(x)=0 \quad \text { on } M \backslash\{o\}, \quad \text { and } \quad g_{p}(x) \asymp \begin{cases}r(x)^{-\frac{m-p}{p-1}} & \text { if } m \neq p, \\ \log 1 / r(x) & \text { if } m=p\end{cases}
$$

as $r(x) \rightarrow 0$. Standard computations then show that

$$
\Delta_{p} g_{p}(x)=\delta_{o}
$$

and therefore $g_{p}$ is a multiple of the radial Green function for $\Delta_{p}$ with pole at $O$.

Proposition 2.9. Let $M$ be a model manifold. Then the following holds

(1) Let $p \leq 2$. If the volume growth condition

$$
\left(\frac{\operatorname{vol} B_{r}(o)}{\operatorname{vol} \partial B_{r}(o)}\right)^{1 /(p-1)} \notin L^{1}(+\infty),
$$

holds, then so does (2.23);

(2) Let $p \geq 2$. If (2.23) holds, then so does (2.25).

Further, none of the reverse implications holds if $p \neq 2$.

Proof. Both the implications follows from suitable applications of the integral Minkowskii inequality.

Assume first that $p \geq 2$. Integrating in polar coordinates and using the explicit expression of $g_{p}$ we have

$$
\begin{aligned}
\int_{M \backslash B_{1}(o)} g_{p}(x)^{p-1} d x & =\int_{1}^{+\infty} d r \sigma(r)^{m-1}\left(\int_{r}^{+\infty} \sigma(t)^{-\frac{m-1}{p-1}} d t\right)^{p-1} \\
& =\left\|\int_{1}^{+\infty}\left(\frac{\sigma(r)}{\sigma(t)} \chi_{[r,+\infty)}(t)\right)^{\frac{m-1}{p-1}} d t\right\|_{L^{p-1}(d r)}^{p-1} \\
& \leq\left\{\int_{1}^{+\infty} d t\left\|\left(\frac{\sigma(r)}{\sigma(t)} \chi_{[1, t)}(r)\right)^{\frac{m-1}{p-1}}\right\|_{L^{p-1}(d r)}\right\}^{p-1} \\
& \leq\left\{\int_{1}^{+\infty} d t\left(\int_{1}^{t} d r \frac{\sigma(r)^{m-1}}{\sigma(t)^{m-1}}\right)^{\frac{1}{p-1}}\right\}^{p-1} \\
& =\left\{\int_{1}^{+\infty} d t\left(\frac{\operatorname{vol} B_{t}-\operatorname{vol} B_{1}}{\operatorname{vol} \partial B_{t}}\right)^{\frac{1}{p-1}}\right\}^{p-1} \\
& \leq\left\{\int_{1}^{+\infty} d t\left(\frac{\operatorname{vol} B_{t}}{\operatorname{vol} \partial B_{t}}\right)^{\frac{1}{p-1}}\right\}^{p-1},
\end{aligned}
$$

showing that (2) holds. 
The proof in case $p \leq 2$ is similar. Now we have $q=\frac{1}{p-1} \geq 1$, and therefore

$$
\begin{aligned}
\int_{M \backslash B_{1}(o)} g_{p}(x)^{p-1} d x & =\int_{1}^{+\infty} d r \sigma(r)^{m-1}\left(\int_{r}^{+\infty}\left[\sigma(t)^{-(m-1)}\right]^{q} d t\right)^{1 / q} \\
& =\int_{1}^{+\infty}\left\|\left(\frac{\sigma(r)}{\sigma(t)} \chi_{[r,+\infty)}(t)\right)^{m-1}\right\|_{L^{q}(d t)} \\
& \geq\left\|\int_{1}^{+\infty} d r\left(\frac{\sigma(r)}{\sigma(t)} \chi_{[1, r)}(r)\right)^{m-1}\right\|_{L^{q}(d t)} \\
& =\left\{\int_{1}^{+\infty} d t\left(\int_{1}^{t} d r \frac{\sigma(r)^{m-1}}{\sigma(t)^{m-1}}\right)^{\frac{1}{p-1}}\right\}^{p-1} \\
& =\left\{\int_{1}^{+\infty} d t\left(\frac{\operatorname{vol} B_{t}-\operatorname{vol} B_{1}}{\operatorname{vol} \partial B_{t}}\right)^{\frac{1}{p-1}}\right\}^{p-1} \\
& \asymp\left\{\int_{1}^{+\infty} d t\left(\frac{\operatorname{vol} B_{t}}{\operatorname{vol} \partial B_{t}}\right)^{\frac{1}{p-1}}\right\}^{p-1},
\end{aligned}
$$

showing that (1) holds.

To see that the reverse implications do not hold if $p \neq 2$, we elaborate on an example of Holopainen, [9]. We let $\sigma$ be such that, for $r \geq 2$

$$
\sigma(r)^{m-1}=r^{-1-\epsilon} \exp \left(r^{q}\right) \quad q=\frac{p+\epsilon}{p-1}
$$

with $\epsilon>0$. An application of de L'Hospital rule shows that, as $r \rightarrow+\infty$

$$
\int_{r}^{+\infty} t^{b} \exp \left(-c t^{a}\right) d t \asymp r^{b-a+1} \exp \left(-C r^{a}\right),
$$

whence, noting that, by the definition of $q, 1-q=-\frac{1+\epsilon}{p-1}$,

$$
g_{p}(x)=\int_{r(x)} t^{\frac{1+\epsilon}{p-1}} \exp \left(-\frac{t^{q}}{p-1}\right) d t \asymp \exp \left(-\frac{r^{q}}{p-1}\right),
$$

and therefore,

$$
\int_{M \backslash B_{1}(o)} g_{p}(x)^{p-1} d x \asymp \int_{1}^{+\infty} t^{-1-\epsilon} d t= \begin{cases}+\infty & \text { if } \epsilon=0 \\ <+\infty & \text { if } \epsilon>0 .\end{cases}
$$

On the other hand,

$$
\operatorname{vol} B_{r}(o)=c_{m} \int_{0}^{r} \sigma(t)^{m-1} d t \asymp t^{-\epsilon-q} \exp \left(t^{q}\right), \quad \text { as } \quad r \rightarrow+\infty,
$$


so that

$$
\left(\frac{\operatorname{vol} B_{r}}{\operatorname{vol} \partial B_{r}}\right)^{1 /(p-1)} \asymp t^{-\frac{1+\epsilon}{(p-1)^{2}}} \quad \text { as } r \rightarrow+\infty
$$

and

$$
\int_{1}^{+\infty}\left(\frac{\operatorname{vol} B_{r}}{\operatorname{vol} \partial B_{r}}\right)^{1 /(p-1)} d t \asymp \int_{1}^{+\infty} t^{-\frac{1+\epsilon}{(p-1)^{2}}}=I(p, \epsilon) .
$$

Now, if $p<2$, then, for every $\epsilon \geq 0, \frac{1+\epsilon}{(p-1)^{2}}>1$ and therefore,

$$
I(p, \epsilon)<+\infty \quad \forall \epsilon \geq 0 .
$$

If $p>2$, then

$$
I(p, \epsilon)= \begin{cases}+\infty & \text { if } 0 \leq \epsilon \leq(p-1)^{2}-1 \\ =+\infty & \text { if } 0 \leq \epsilon>(p-1)^{2}-1\end{cases}
$$

Thus, if $p<2$, choosing $\epsilon=0$, we have

$$
\int_{1}^{+\infty} g_{p}(x)^{p-1} d x=+\infty \text { and } \int_{1}^{+\infty}\left(\frac{\operatorname{vol} B_{t}}{\operatorname{vol} \partial B_{t}}\right)^{\frac{1}{p-1}} d t<+\infty,
$$

showing that the reverse implication does not hold in (1).

Similarly, if $p>2$, choosing $0<\epsilon \leq(p-1)^{2}-1$, we have

$$
\int_{1}^{+\infty}\left(\frac{\operatorname{vol} B_{t}}{\operatorname{vol} \partial B_{t}}\right)^{\frac{1}{p-1}} d t=+\infty, \quad \text { and } \quad \int_{1}^{+\infty} g_{p}(x)^{p-1} d x=+\infty,
$$

showing that the reverse implication does not hold in (2).

Remark 2.10. In the above example, one has simultaneously

$$
\int_{M \backslash B_{1}(o)} g_{p}(x)^{p-1} d x=+\infty \text { and } \int_{1}^{+\infty}\left(\frac{t}{\log \operatorname{vol} B_{t}}\right)^{p-1} d t=+\infty(\epsilon=0),
$$

or

$$
\int_{M \backslash B_{1}(o)} g_{p}(x)^{p-1} d x<+\infty \text { and } \int_{1}^{+\infty}\left(\frac{t}{\log \operatorname{vol} B_{t}}\right)^{p-1} d t<+\infty(\epsilon>0) .
$$

This led Holopainen to conjecture that the $L^{p-1}$-Liouville property is implied by (equivalent to) the volume growth condition

$$
\int_{1}^{+\infty}\left(\frac{t}{\log \operatorname{vol} B_{t}}\right)^{p-1} d t=+\infty
$$


In this respect, observe that standard arguments (see, e.g. [23, Proposition 1.3]) show that, if $\operatorname{vol} B_{t} \nearrow+\infty$, then for every $\beta>0$

$$
\int_{1}^{+\infty}\left(\frac{\operatorname{vol} B_{t}}{\operatorname{vol} \partial B_{t}}\right)^{\beta} d t \geq C \int_{1}^{+\infty}\left(\frac{t}{\log \operatorname{vol} B_{t}}\right)^{\beta} d t
$$

so that one is led to compare the divergence of the following two integrals

$$
\int_{1}^{+\infty}\left(\frac{t}{\log \operatorname{vol} B_{t}}\right)^{1 /(p-1)} d t \text { and } \int_{1}^{+\infty}\left(\frac{t}{\log \operatorname{vol} B_{t}}\right)^{p-1} d t
$$

While Proposition 2.9 provides circumstantial evidence that the divergence of the first integral is relevant to the $L^{p-1}$-Liouville property, in all the examples where the authors have been able to carry out the computations the integral

$$
\int_{M \backslash B_{1}(o)} g_{p}(x)^{p-1} d x
$$

turned out to diverge exactly when the so did the second integral in (2.33).

The search for the correct geometric properties implying the validity of the $L^{p-1}$-Liouville property suggests other interesting problems.

By way of example, observe that while the stochastic completeness of a manifold is not invariant under quasi isometries (see [15]), conditions involving the divergence of the integral of powers of $\frac{t}{\log \operatorname{vol} B_{t}}$ clearly are invariant under quasi-isometries. One is therefore led to ask the question if the $L^{p-1}$ Liouville property is invariant under quasi-isometries. To the authors' best knowledge, the answer to this question is still not known.

Yet, the category of (non-parabolic) manifolds supporting a global elliptic Harnack inequality gives some indications. Indeed, let $(M,\langle\rangle$,$) be a$ manifold in this category. Let $\rho(x)$ be a smooth exhaustion function (i.e., its level sets are relatively compact and $\rho$ diverges at infinity), and denote by $\Omega_{t}$ the level set $\{x: \rho(x)<t\}$. We choose a reference point $o$ in $\Omega_{1}$ and use Sard's theorem to find a diverging sequence $t_{k}$ with $t_{1}>1$ such that $\Omega_{t_{k}}$ has smooth boundary.

An argument of Holopainen, see Theorem 3.19 in [8], shows that the Dirichlet, $p$-Green function $g_{k}$ of $\Omega_{t_{k}}$ with pole at $o$ satisfies

$$
\lambda^{-1} \operatorname{cap}_{p}\left(\bar{\Omega}_{t}, \Omega_{t_{k}}\right)^{1 /(1-p)} \leq g_{k} \leq \lambda \operatorname{cap}_{p}\left(\bar{\Omega}_{t}, \Omega_{t_{k}}\right)^{1 /(1-p)} \text { on } \partial \Omega_{t}
$$

for every $t \in\left[1, t_{k}\right)$. Here $\lambda>1$ is the global Harnack constant of $(M,\langle\rangle$, and $\operatorname{cap}_{p}\left(\bar{\Omega}_{t}, \Omega_{t_{k}}\right)$ is the $p$-capacity of the condenser $\left(\bar{\Omega}_{t}, \Omega_{t_{k}}\right)$ which is variationally defined as

$$
\operatorname{cap}_{p}\left(\bar{\Omega}_{t}, \Omega_{t_{k}}\right)=\inf \left\{\int_{M}|\nabla \varphi|^{p}: \varphi \in C_{0}^{\infty}\left(\Omega_{t_{k}}\right), \varphi \equiv 1 \text { on } \bar{\Omega}_{t}\right\} .
$$


Letting $k \rightarrow+\infty$, the sequence $\left\{g_{k}\right\}$ converges, locally uniformly on $M$, to the $p$-Green function $g$ of $(M,\langle\rangle$,$) with pole o$ and $\operatorname{cap}_{p}\left(\bar{\Omega}_{t}, \Omega_{t_{k}}\right)$ converges to the absolute capacity $\operatorname{cap}_{p}\left(\bar{\Omega}_{t}\right)$ and from $(2.34)$ we deduce

$$
\lambda^{-1} \operatorname{cap}_{p}\left(\bar{\Omega}_{t}\right)^{1 /(1-p)} \leq g \leq \lambda \operatorname{cap}_{p}\left(\bar{\Omega}_{t}\right)^{1 /(1-p)} \quad \text { on } \partial \Omega_{t}
$$

for every $t \geq 1$. Now, suppose $M$ is endowed with a second (non-parabolic) metric $\widetilde{\langle,\rangle}$ satisfying a global Harnack inequality. Denoting quantities related to this metric with a tilde ${ }^{\sim}$, with the obvious meaning of symbols we have

$$
\tilde{\lambda}^{-1} \widetilde{\operatorname{cap}}_{p}\left(\bar{\Omega}_{t}\right)^{1 /(1-p)} \leq \tilde{g} \leq \tilde{\lambda} \widetilde{\operatorname{cap}}_{p}\left(\bar{\Omega}_{t}\right)^{1 /(1-p)} \quad \text { on } \partial \Omega_{t}
$$

for every $t \geq 1$. If we assume that $\langle$,$\rangle and \widetilde{\langle,\rangle}$ are quasi isometric then, according to (2.35), we have the further relations

$$
C_{1}^{-1} \widetilde{\operatorname{cap}}_{p}\left(\bar{\Omega}_{t}\right) \leq \operatorname{cap}_{p}\left(\bar{\Omega}_{t}\right) \leq C_{1} \widetilde{\operatorname{cap}}_{p}\left(\bar{\Omega}_{t}\right)
$$

for some absolute constant $C_{1}>1$. Combining (2.36), (2.37) and (2.38) we therefore conclude that, for some absolute constant $C_{2}>1$,

$$
C_{2}^{-1} \tilde{g} \leq g \leq C_{2} \tilde{g} \quad \text { on } M-\Omega_{1}
$$

proving that $(M,\langle\rangle$,$) is (p-1)$-Liouville if and only if so is $(M, \widetilde{\langle,\rangle})$.

Acknowledgements. The authors are grateful to Ilkka Holopainen for several useful conversations on the subject of the paper and for calling their attention to the $L^{p-1}$-Liouville property.

\section{References}

[1] Cheeger, J., Gromov, M. And Taylor, M.: Finite propagation speed, kernel estimates for functions of the Laplace operator, and the geometry of complete Riemannian manifolds. J. Differential Geom. 17 (1982), 15-53.

[2] Coulhon, T., Holopainen, I. and Saloff-Coste, L.: Harnack inequality and hyperbolicity for subelliptic $p$-Laplacians with applications to Picard type theorems. Geom. Funct. Anal. 11 (2001), 1139-1191.

[3] Díaz, J. I.: Nonlinear partial differential equations and free boundaries. Vol I. Elliptic equations. Research Notes in Mathematics 106. Pitman, Boston, MA, 1985.

[4] Greene, R. E. And Wu, H.: Function theory on manifolds which possess a pole. Lecture Notes in Mathematics 699. Springer, Berlin, 1979. 
[5] Grigor'yan, A.: Analytic and geometric background of recurrence and non-explosion of the Brownian motion on Riemannian manifolds. Bull. Amer. Math. Soc. (N.S.) 36 (1999), 135-249.

[6] Grigor'yan, A.: Stochastically complete manifolds and summable harmonic functions. (Russian) Izv. Akad. Nauk SSSR Ser. Mat. 52 (1988), 1102-1108; translation in Math. USSR-Izv. 33 (1989), 425-432.

[7] Heinonen, J., KilpeläInen, T. And Martio, O.: Nonlinear potential theory of degenerate elliptic equations. Oxford Mathematical Monographs. Oxford Science Publications. The Clarendon Press, Oxford University Press, New York, 1993.

[8] Holopainen, I.: Nonlinear potential theory and quasiregular mappings of Riemannian manifolds. Ann. Acad. Sci. Fenn. Ser. A I Math. Dissertationes 74 (1990), 1-45.

[9] Holopainen, I.: A sharp $L^{q}$-Liouville theorem for $p$-harmonic functions. Israel J. Math. 115 (2000), 363-379.

[10] Holopainen, I.: Volume growth, Green's functions, and parabolicity of ends. Duke Math. J. 97 (1999), 319-346.

[11] Kazdan, J. L. And Warner, F. W.: Curvature functions for open 2manifolds. Ann. of Math. (2) 99 (1974), 203-219.

[12] KuRA, T.: The weak supersolution-subsolution method for second order quasilinear elliptic equations. Hiroshima Math. J. 19 (1989), 1-36.

[13] Kuramochi, Z.: Mass distributions on the ideal boundaries of abstract Riemann surfaces. I. Osaka Math. J. 8 (1956), 119-137.

[14] LE, V.K.: On some equivalent properties of sub- and supersolutions in second order quasilinear elliptic equations. Hiroshima Math. J. 28 (1998), 373-380.

[15] LyOns, T.: Instability of the conservative property under quasi-isometries. J. Differential Geom. 34 (1991), 483-489.

[16] Nakai, M.: On Evans potentials. Proc. Japan Acad. 38 (1962), 624-629.

[17] Pigola, S., Rigoli, M. And Setti, A. G.: A remark on the maximum principle and stochastic completeness. Proc. Amer. Math. Soc. 131 (2003), $1283-1288$.

[18] Pigola, S., Rigoli, M. and Setti, A. G.: Maximum principles on Riemannian manifolds and applications. Mem. Amer. Math. Soc. 174 (2005).

[19] Pucci, P. And Serrin, J.: A note on the strong maximum principle for elliptic differential inequalities. J. Math. Pures Appl. (9) 79 (2000), 57-71.

[20] Pucci, P. And Serrin, J.: The strong maximum principle revisited. J. Differential Equations 196 (2004), 1-66.

[21] Pucci, P., Serrin, J. And Zou, H.: A strong maximum principle and a compact support principle for singular elliptic inequalities. J. Math. Pures Appl. (9) $\mathbf{7 8}$ (1999), 769-789. 
[22] Ratto, A., Rigoli, M. and Setti, A. G.: On the Omori-Yau maximum principle and its application to differential equations and geometry. J. Funct. Anal. 134 (1995), 486-510.

[23] Rigoli, M. and Setti, A. G.: Liouville type theorems for $\varphi$-subharmonic functions. Rev. Mat. Iberoamericana 17 (2001), 471-520.

[24] Sario, L. ANd NAKAI, M.: Classification theory of Riemann surfaces. Die Grundlehren der mathematischen Wissenschaften 164. Springer-Verlag, New York-Berlin 1970.

[25] Serrin, J. And Zou, H.: Cauchy-Liouville and universal boundedness theorems for quasilinear elliptic equations and inequalities. Acta Math. 189 (2002), 79-142.

[26] Tolksdorf, P.: Regularity of a more general class of quasilinear elliptic equations. J. Differential Equations 51 (1984), 126-150.

[27] VÁzquez, J. L.: A strong maximum principle for some quasilinear elliptic equations. Appl. Math. Optim. 12 (1984), 191-202.

[28] YAU, S. T.: Some function-theoretic properties of complete Riemannian manifolds and their applications to geometry. Indiana Univ. Math. J. 25 (1976), 659-670.

Recibido: 15 de abril de 2004

Stefano Pigola

Dipartimento di Fisica e Matematica

Università dell'Insubria-Como

via Valleggio 11

I-22100 Como, Italy

stefano.pigola@uninsubria.it

Marco Rigoli

Dipartimento di Matematica

Università di Milano

via Saldini 50

I-20133 Milano, Italy

rigoli@mat . unimi.it

Alberto G. Setti

Dipartimento di Fisica e Matematica

Università dell'Insubria-Como

via Valleggio 11

I-22100 Como, Italy

alberto.setti@uninsubria.it 